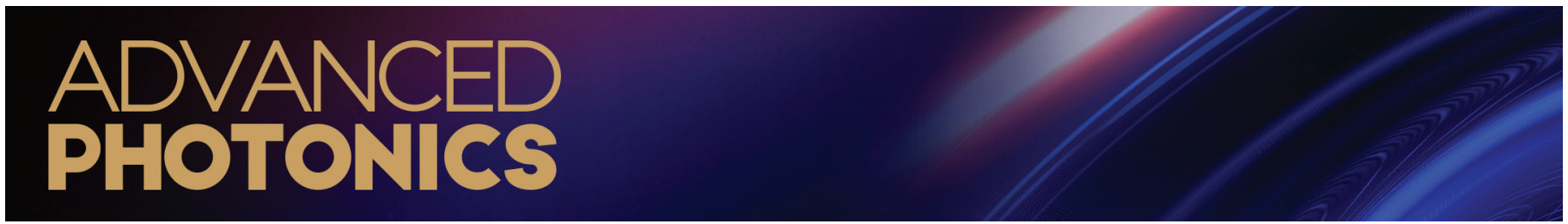

\title{
Optical superoscillatory waves without side lobes along a symmetric cut
}

\author{
Yanwen Hu, ${ }^{a}$ Shiwang Wang, ${ }^{a}$ Junhui Jia, ${ }^{a}$ Shenhe $\mathrm{Fu}^{\mathrm{a}, \mathrm{b}, \mathrm{b}}$ Hao Yin, ${ }^{\mathrm{a}, \mathrm{b}}$ Zhen Li, ${ }^{\mathrm{a}, \mathrm{b}, \star}$ and Zhenqiang Chen ${ }^{\mathrm{a}, \mathrm{b}}$ \\ ajinan University, Department of Optoelectronic Engineering, Guangzhou, China \\ ${ }^{b}$ Guangdong Provincial Key Laboratory of Optical Fiber Sensing and Communications, Guangzhou, China
}

\begin{abstract}
Optical superoscillation refers to an intriguing phenomenon of a wave packet that can oscillate locally faster than its highest Fourier component, which potentially produces an extremely localized wave in the far field. It provides an alternative way to overcome the diffraction limit, hence improving the resolution of an optical microscopy system. However, the optical superoscillatory waves are inevitably accompanied by strong side lobes, which limits their fields of view and, hence, potential applications. Here, we report both experimentally and theoretically a new superoscillatory wave form, which not only produces significant feature size down to deep subwavelength, but also completely eliminates side lobes in a particular dimension. We demonstrate a new mechanism for achieving such a wave form based on a pair of moonlike sharp-edge apertures. The resultant superoscillatory wave exhibits Bessel-like forms, hence allowing long-distance propagation of subwavelength structures. The result facilitates the study of optical superoscillation and on a fundamental level eliminates the compromise between the superoscillatory feature size and the field of view.
\end{abstract}

Keywords: optical superoscillation; wave propagation; sharp-edge diffraction.

Received May 30, 2020; revised manuscript received May 3, 2021; accepted for publication May 28, 2021; published online Jun. 23, 2021.

(C) The Authors. Published by SPIE and CLP under a Creative Commons Attribution 4.0 Unported License. Distribution or reproduction of this work in whole or in part requires full attribution of the original publication, including its DOI.

[DOI: 10.1117/1.AP.3.4.045002]

\section{Introduction}

Owing to the wave nature of optical diffraction, it is extremely challenging to achieve a highly localized wave packet in free space, which essentially limits the imaging performance of an optical system. In 1873, Erns Abbe first introduced the concept of optical diffraction limit, ${ }^{1}$ which is a constraint on the smallest light spot with a spatial size of about $\lambda / 2$, where $\lambda$ is the optical wavelength. Essentially, the diffractive light wave with high spatial frequency, named an evanescent wave, tends to exist on the near-field surface of an object and decays exponentially with distance. As a result, delicate information of the object carried by the evanescent wave cannot be delivered to the far-field region. In the past, substantial effort was made to break such a diffraction barrier ${ }^{2-4}$ so as to achieve higher spatial resolution. ${ }^{25,6}$ The earlier developed techniques were mainly focused on exploiting high-spatial-frequency evanescent waves with a nanotip, ${ }^{7,8}$ superlens, ${ }^{9-11}$ or hyperlens. ${ }^{12}$ But, these

${ }^{*}$ Address all correspondence to Shenhe Fu, fushenhe@jnu.edu.cn; Zhen Li, ailz268@126.com methods encounter serious limitations of near-field manipulations or unattainable fabrications. Later on, different fluorescent labeling technologies were proposed to overcome the diffraction barrier in the far field. ${ }^{5,13,14}$ Despite their ultrahigh resolving property, these techniques depend on fluorescent labeling and require careful calibration with specific dye molecules.

A promising technique is to explore optical superoscillatory waves in the far field ${ }^{15-18}$ instead of the evanescent waves in the near field. In this situation, Abbe's diffraction limit can be discarded to allow superresolution by virtue of creating a localized spot of a focused light beam, while the beam's overall width is wider than Abbe's limit. For example, it has been reported that performing confocal microscopy with superoscillation by structuring the higher-order vector beams ${ }^{19}$ or by carefully tailoring coherent superposition of diffracted waves from a nanostructured mask ${ }^{16}$ can achieve a local light spot below the diffraction limit. Indeed, superoscillation is an intriguing phenomenon of a bandlimited function that can oscillate at a frequency exceeding its highest Fourier component. ${ }^{20}$ It was examined previously in the field of quantum mechanics as well as in general signal theory. ${ }^{21}$ Recently, Berry and Popescu ${ }^{20}$ introduced the concept 
of superoscillation in optics and suggested potential applications of optical superoscillatory waves in far-field superresolution imaging without the evanescent waves. ${ }^{15,20}$ Subsequently, the predication was experimentally verified by designing nanostructured masks, named superoscillatory lenses (SOLs). ${ }^{16,22-26}$ However, these particular SOLs might encounter issues: first, the design of the SOLs relies excessively on optimization algorithms, which poses great challenges in mask fabrications; second, the generated superoscillatory light wave is accompanied by strong side lobe, which limits the field of view. These disadvantages of complex structural design and huge side lobe near the superoscillatory region affect its practical applications. ${ }^{16}$ Effort has been invested to constrain the side lobe using a set of rings placed at appropriate positions, ${ }^{27}$ but it is necessary to precisely tailor the dimension and the transmission of each ring, which poses many difficulties of a practical nature, or by designing complex type of planar supercritical lens, ${ }^{28}$ but it comes necessarily at the cost of enlarging the main lobe. Owing to the compromise, up till now, it has remained challenging to design an easy-to-perform structure that can produce superoscillatory waves with appreciable feature size while maintaining a large field of view.

In this article, we introduce both experimentally and theoretically a new form of optical superoscillatory wave, which eliminates the tradeoff between the superoscillatory feature size and the field of view. We note that the creation of the optical superoscillatory wave is inevitably accompanied by the side lobe around the main lobe. ${ }^{15,16,19,29-33}$ We present a mechanism based on a pair of moonlike apertures to overcome this issue. Such moonlike elements allow not only generating superoscillatory light waves in the far field but also eliminating completely their side lobes along a symmetric cut of the wave patterns. In comparison to the optimization-based generation, ${ }^{16,22-26}$ the proposed principle has remarkable advantages: easy-to-perform, optimization-free, and robust to the changes of light parameters such as wavelength and polarization. In addition, this work also addresses propagation of the generated superoscillatory waves, featured by their weakly diffracting property in free space. The generated superoscillatory light wave holds potential applications, e.g., it can be utilized to enhance light-matter interaction in the nanoscale, as well as in the field of superresolution imaging.

\section{Principle}

The superoscillatory wave is produced from constructive interference of many plane wave components having high spatial frequency. These components should be in phase, i.e., their wave vectors exhibit cylindrically symmetric distribution in reciprocal space. To achieve this, a basic circular disc $C_{1}$ is required, as shown by step 1 in Fig. 1(a), with diameter $d$. Mathematically, the basic circular disc can be written in a form of $C_{1}=1-\operatorname{circ}\left[\sqrt{x^{2}+y^{2}} /(d / 2)\right]$, where $\operatorname{circ}(\cdot)$ represents the circle function, and $x, y$ are the spatial coordinates. Inside the circle, $\operatorname{circ}(\cdot)=1$, whereas outside the $\operatorname{circle} \operatorname{circ}(\cdot)=0$. A superoscillatory hotspot is expected behind the disc, accompanied by strong side waves that come from the zero-order diffraction. To eliminate the side waves, a destructive interference for them is considered. For this reason, we shift $C_{1}$ horizontally to the right (see the step 2) and to the left (see the step 3) by a distance of $\omega$, to obtain the other two circular elements of $C_{2}$ and $C_{3}$, respectively. Unlike the circular disc function, $C_{2}$ and $C_{3}$ have a form of circular aperture function, expressed as $C_{2}=$ $\operatorname{circ}\left[\sqrt{(x-\omega)^{2}+y^{2}} /(d / 2)\right]$ and $C_{3}=\operatorname{circ}\left[\sqrt{(x+\omega)^{2}+y^{2}} /\right.$ $(d / 2)]$, respectively. Clearly, $C_{2}$ and $C_{3}$ are symmetric with respect to the $y$ axis. To display the moonlike structure explicitly, we erase the parts of $C_{2}$ and $C_{3}$ that overlap with the basic circular disc $C_{1}$, as illustrated in step 4 . Since $C_{2}$ and $C_{3}$ deviate a little bit from the original position of $C_{1}$ to opposite directions, we also remove the nonoverlapping curves of $C_{1}$ with $C_{2}$ and $C_{3}$; see the top and the bottom of the structure in step 5. Finally, we obtain two moonlike apertures having symmetric geometry with respect to the $y$ axis, as shown in the final step 6. It is seen that the symmetric cut of the structure leads to two disconnected apertures and creates a dark line along the $y$ coordinate, and the line width depends on the displacement $\omega$. It is clear that the nanostructure is slightly symmetry breaking with respect to the cylindrical coordinate system, which leads to a destructive interference effect of diffractive waves along the line perpendicular to the apertures. We further describe such a pair of apertures with a general formula, given by $C=C_{1}\left(C_{2}+C_{3}\right)$, i.e.,

$$
\begin{aligned}
C(x, y)= & {\left[1-\operatorname{circ}\left(\frac{\sqrt{x^{2}+y^{2}}}{d / 2}\right)\right] \times\left[\operatorname{circ}\left(\frac{\sqrt{(x-\omega)^{2}+y^{2}}}{d / 2}\right)\right.} \\
& \left.+\operatorname{circ}\left(\frac{\sqrt{(x+\omega)^{2}+y^{2}}}{d / 2}\right)\right]
\end{aligned}
$$

Obviously, the obtained moonlike apertures could be treated with two critical parameters, which are the diameter $d$ of the basic circular disc and the horizontal displacement $\omega$, which determines the width of each moonlike aperture.

When an optical plane wave passes through the structure, its incident amplitude $E_{0}$ would be modulated in binary. Namely, the incident light field equals the emerging field at the output side of the apertures, while outside the apertures the amplitude becomes zero, which can be expressed as $E_{0} \times C(x, y)$. In this scenario, the amplitude near the edge has a form of step function, which exhibits sharp-edged optical diffraction near the edge and hence generates significant high-spatial-frequency wavevectors in its reciprocal space. ${ }^{34,35}$ As a result, the incident light wave is diffracted seriously while propagating through the apertures. On the other hand, since the two symmetric moonlike apertures are separated by the basic circular disc, as shown in the final step in Fig. 1(a), there exhibits, in the vicinity of the circular disc, a circular step function that processes abundant high-order frequency components having cylindrical distribution in the reciprocal space. As a consequence, the in-phase high-spatial-frequency wavevectors point to identical central positions behind disc $C_{1}$, generating a constructive light wave (a phenomenon of the Poisson-Arago spot) with the transverse size expectantly beyond the diffraction limit.

To experimentally generate such a superoscillatory light wave, a critical issue is to realize binary amplitude modulation of the incident light waves with the designed moonlike apertures. Thus, it requires that the amplitude of the incident plane wave jumps sharply at the edge of each moonlike aperture. We achieve this goal using an extremely thin metallic film deposited on a glass substrate. Specifically, a gold film whose thickness is only $50 \mathrm{~nm}$ was initially deposited onto the 0.3 -mm-thick substrate. A 10-nm-thick chromium film was deposited between an adhesion layer. We used UV lithography to form a mask, and finally we used the ion beam to etch the mask to obtain the final 
(a)

step 1

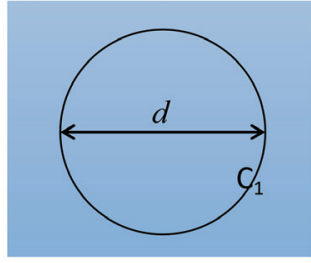

step 6

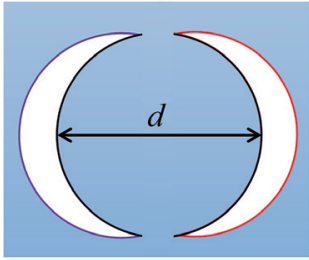

(d)

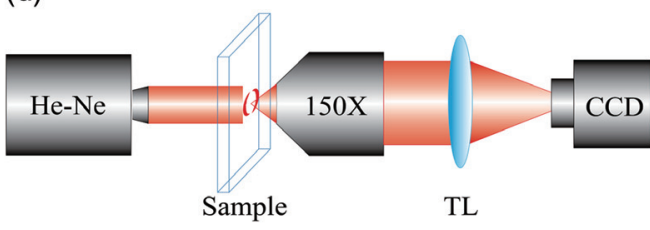

step 2

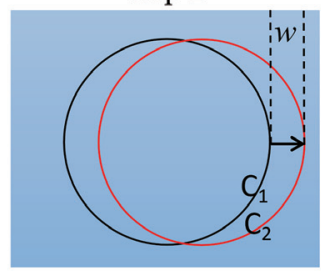

step 5

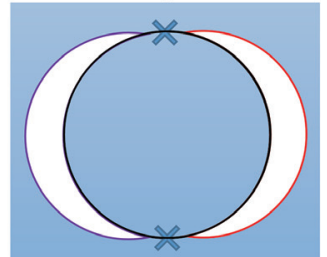

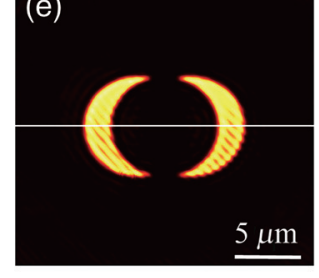

step 3

step 4

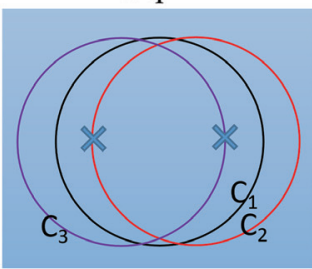

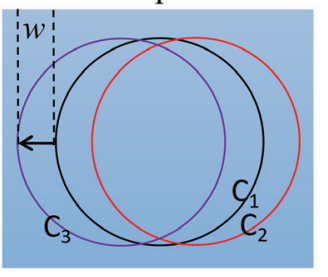
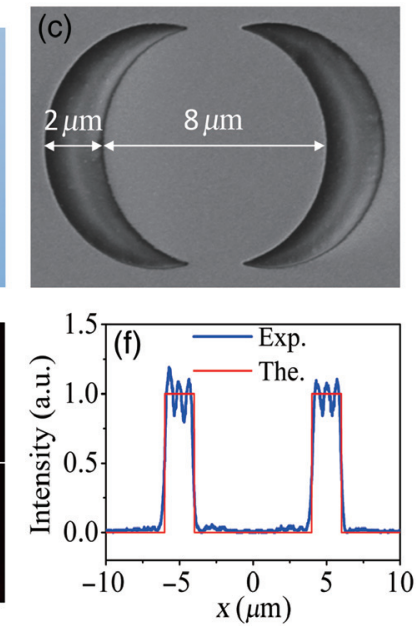

Fig. 1 Principle for generating the no-side-lobe optical superoscillatory waves with a pair of symmetric moonlike apertures. (a) Design processes for the moonlike apertures. (b) The moonlike apertures were fabricated with an extremely thin gold film deposited on a glass substrate. (c) An electron micrograph of one of the fabricated samples. (d) Experimental setup used to validate the binary amplitude modulation of the moonlike apertures as well as to measure the diffractive waves in the far field with an He-Ne laser working at a wavelength of $\lambda=633 \mathrm{~nm}$. (e), (f) Initial measurement of the output field from the sample: (e) intensity distribution in the $x-y$ plane; (f) cross-section of the light field as marked with a white line in (e). The blue curve denotes the experiment, while the red curve shows the theory.

moonlike patterns, as shown in Fig. 1(b). Two metallic moonlike structures with typical parameters $d=8 \mu \mathrm{m}, \omega=2 \mu \mathrm{m}$ and $d=15 \mu \mathrm{m}, \omega=2 \mu \mathrm{m}$ were simultaneously fabricated on the same substrate. Figure 1(c) shows an electron micrograph of one of the fabricated moonlike apertures. Compared to the optical wavelength and the sample size, the thickness of the samples is ignorable. Therefore, we assume that the wave fields emerging from the apertures could be described by the Kirchhoff boundary conditions.

An experimental setup, as shown in Fig. 1(d), was used to verify the assumption. A linearly polarized He-Ne laser working at a wavelength of $\lambda=633 \mathrm{~nm}$ was collimated, and then the moonlike sample was illuminated from the substrate side. To measure the output light field from the sample that is placed at position $z=0$, where $z$ denotes the propagation distance of the waves, we utilized an objective lens with high magnification (Nikon, CFI EPI $150 \times$, numerical aperture NA $=0.9$ ) together with a tube lens (TL) to image the output field onto a charge coupled device (CCD) with pixel size of $1.4 \mu \mathrm{m}$. With such a configuration, we initially characterize the binary amplitude modulation at the edge of the moonlike apertures (as an example, here we only discuss the sample with parameters: $d=8 \mu \mathrm{m}$ and $\omega=2 \mu \mathrm{m}$ ). Figure 1(e) shows intensity distribution of the output field in the $x-y$ plane. Clearly, the intensity of the light field inside the apertures becomes nearly invariant, while outside the aperture the field is blocked. It indicates that a step function of the amplitude at the edge is approximately exhibited, which is further confirmed by a selected intensity profile (at $y=0$ ) along the $x$ coordinate, as shown in Fig. 1(f). The initial measurement shows good agreement with the theoretical result and validates approximately the Kirchhoff boundary conditions.

\section{Results and Discussion}

To observe the superoscillatory light waves, we carefully moved the objective lens to measure the diffractive light fields at different propagation distances. In this case, the objective lens was mounted on an electrical-control stage (MMC-100/PPX-32) with spatial resolution in the $z$-axis being $20 \mathrm{~nm}$. Figure 2 shows the measured intensity distributions of the light fields at distances of $z=4.9 \mu \mathrm{m}, z=5.5 \mu \mathrm{m}$, and $z=6.2 \mu \mathrm{m}$, corresponding to Figs. 2(a)-2(c), respectively. From the results, we observe that, in addition to the strong intensity inside the aperture region, the diffractive waves exhibit an extremely localized main lobe in the center behind disc $C_{1}$. The observed super diffractive focusing light spot confirms the constructive superposition of the in-phase diffracted waves that have higher-spatial-frequency wavevectors than the original one, $k_{0}$, where $k_{0}=2 \pi / \lambda$. Moreover, it is intriguing to observe that the generated superoscillatory main lobe has no side lobe accompanied along 

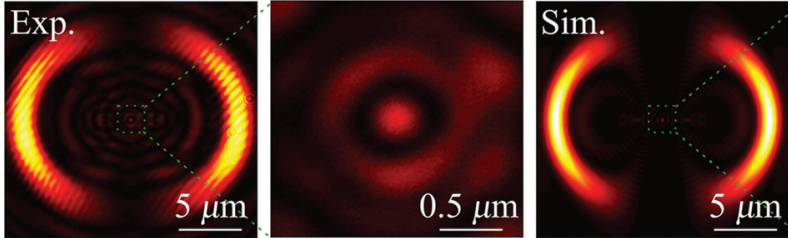

(a)
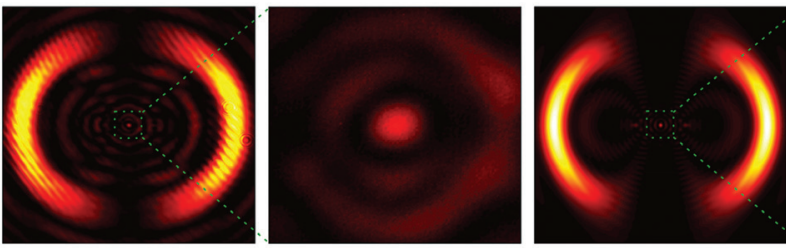

(b)
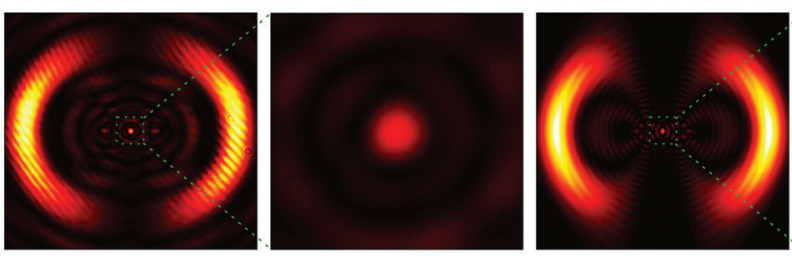

(c)
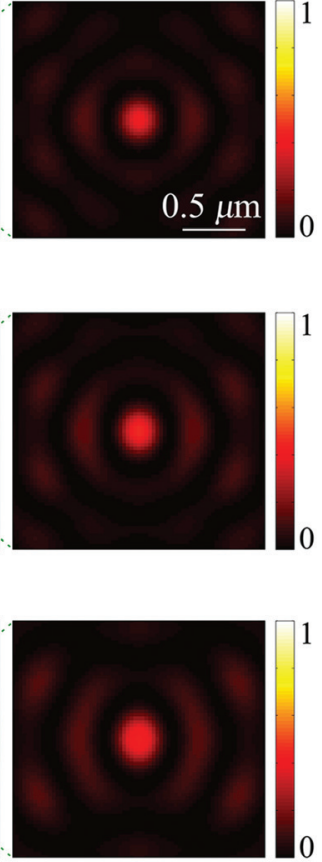

Fig. 2 Experimental and numerical demonstrations of the generated superoscillatory light waves with the moonlike nanostructure (parameters: $d=15 \mu \mathrm{m}$ and $\omega=2 \mu \mathrm{m}$ ). (a)-(c) The intensity distributions of the diffractive light waves in the transverse plane at different distances: (a) $z=4.9 \mu \mathrm{m}$, (b) $z=5.5 \mu \mathrm{m}$, and (c) $z=6.2 \mu \mathrm{m}$. The panels in the second and the fourth columns are the zoom-in intensity distributions. Panels in each column share the same scale. The wavelength used here is $\lambda=633 \mathrm{~nm}$.

the symmetric cut. Such a no-side-lobe feature of the focusing light spot is caused by a destructive interference of diffracted waves along the line perpendicular to the apertures. To further characterize the superoscillatory behavior, their corresponding zoom-in intensity distributions are also presented, see Fig. 2, as well as their intensity profiles along the $x$ and $y$ coordinates, as shown in Figs. 3(a), 3(c), 3(e) and 3(b), 3(d), 3(f), respectively. The full-width at half-maximum (FWHM) of the main lobe along the $y$ coordinate was measured as $214 \mathrm{~nm}$ $(\sim \lambda / 3), 230 \mathrm{~nm}(\sim \lambda / 2.7)$, and $285 \mathrm{~nm}(\sim \lambda / 2.2)$, respectively, all below the optical diffraction limit. Owing to the destructive interference effect, these superoscillatory spots at different distances exhibit almost no side waves, which breaks the compromise between the superoscillatory main lobes and the side lobes. It is further observed that the superoscillatory spot size slightly increases with the diffractive distance. On the other hand, the superoscillatory main lobes along $x$ exhibit a similar feature size at the corresponding distance, which further confirms the cylindrical wave diffraction from the central disc $C_{1}$. Contrary to the intensity profile along the $y$ axis, the generated light spot along the $x$ axis is surrounded by significant side waves.

To confirm the experimental results, we investigate theoretically the diffractive property of the moonlike structure. Using the Rayleigh-Somerfield theory ${ }^{36}$ the diffractive light field beyond the structure $(z>0)$ can be calculated as follows:

$E(x, y, z)=\frac{E_{0}}{i \lambda} \iint C\left(x^{\prime}, y^{\prime}\right) \frac{z}{p^{2}} \exp \left(i k_{0} p\right) \mathrm{d} x^{\prime} \mathrm{d} y^{\prime}$, where $E_{0}$ denotes the amplitude of the incident plane wave, and

$p=\left[\left(x-x^{\prime}\right)^{2}+\left(y-y^{\prime}\right)^{2}+z^{2}\right]^{\frac{1}{2}}$.

To achieve the optical superoscillatory waves with a symmetric cut, we adopted the same parameters as those used in the experiments. With these conditions, we produced the simulated outcomes shown in Fig. 2, as well as their corresponding intensity profiles; see the red curves in Fig. 3. They clearly show the no-side-lobe feature of the superoscillatory waves along the $y$ axis. Both the intensity distributions and the cross sections at the selected region approximately reproduce the experimental results.

We further evaluate the focusing efficiency of the nanostructure. This can be done by using the ratio of peak intensity in the main lobe and the strongest side lobe. ${ }^{29,37,38}$ In theory, the peak intensity of the superoscillatory main lobe in Fig. 2(b) can reach $27.8 \%$ of that in the strongest side lobe, whereas in the experiment it is measured as $25.4 \%$, further confirming the validity of the theoretical model in Eq. (2). Another way to characterize the focusing efficiency is by using the ratio of light power in the central hotspot and the input light field. ${ }^{39,40}$ In this scenario, the efficiency is usually much lower, since the integral for the light power is just over the superoscillatory area. For example, considering the result shown in Fig. 2(b), the resultant efficiency (in theory) is $0.74 \%$. The focusing efficiency obtained here is comparable to previous superoscillatory techniques..$^{29,37-40}$ The efficiency can be relatively enhanced (lowered) when increasing 


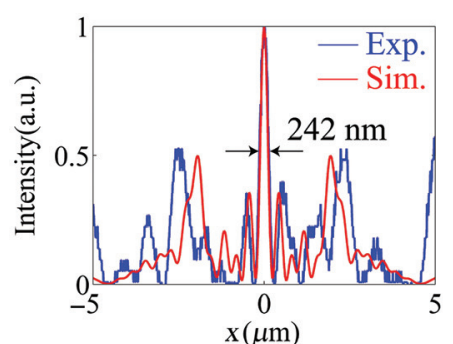

(a)

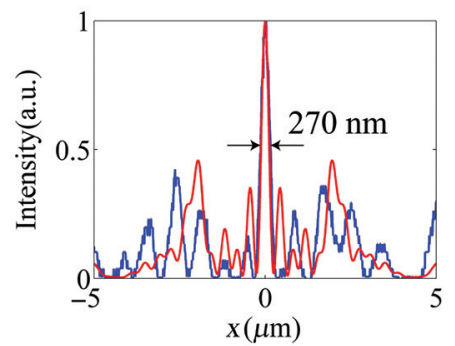

(c)

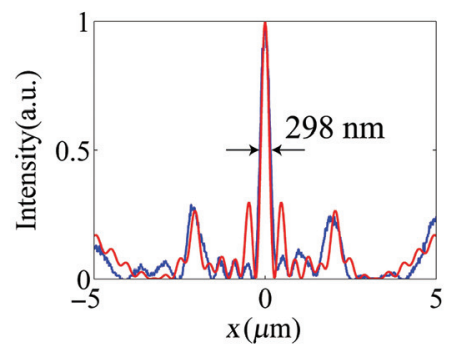

(e)

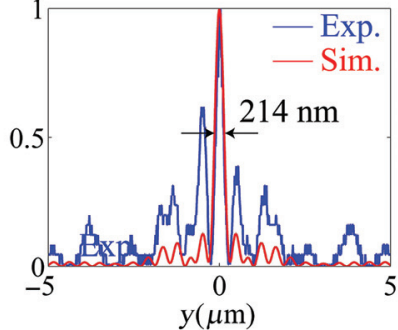

(b)

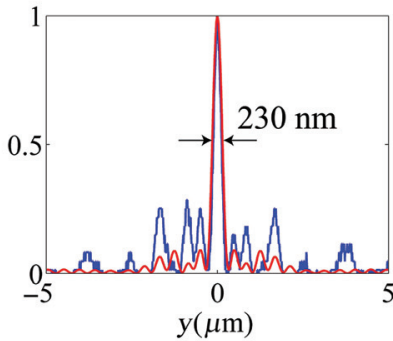

(d)

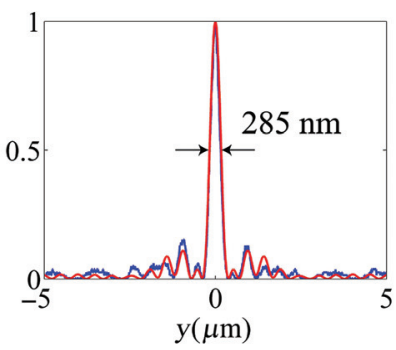

(f)

Fig. 3 The intensity cross-sections of the superoscillatory lobes at different distances: (a), (b) $z=4.9 \mu \mathrm{m}$; (c), (d) $z=5.5 \mu \mathrm{m}$; and (e), (f) $z=6.2 \mu \mathrm{m}$. (a), (c), (e) The intensity profiles along the $x$ axis $(y=0)$; (b), (d), (f) the intensity profiles along the $y$ axis $(x=0)$. The corresponding FWHMs were indicated experimentally in the panels. The blue curves represent the experiments, while the red curves denote the simulations based on Eq. (2). The wavelength used here is $\lambda=633 \mathrm{~nm}$.

(decreasing) the propagation distance, accompanied by a relatively larger (smaller) superoscillatory spot size.

Note that a slight deviation between the experimental side lobes and the simulated outcomes is shown in Fig. 3. This difference stems from the approximate fulfillment of the binary modulation at the edge of the sample. As shown in Fig. 1(f), the imperfect binary amplitude modulation together with the nonuniform incident amplitude [see slight intensity oscillations in Fig. 1(f)] might contribute to generating uncertain side lobes in the experiments. These imperfect experimental conditions increase difficulties in observing the minor asymmetric distribution of the superoscillatory main lobe, as shown in the simulations in Fig. 2. Moreover, the detection sensitivity of the CCD camera used here is insufficient to capture the weak intensity of the superoscillatory side lobes, leading to low signal-to-noise ratio, as shown in Figs. 3(a) and 3(b). However, this could be improved when the superoscillatory main lobe becomes stronger, as illustrated from the measurements in Figs. 3(e) and 3(f), respectively.

In the following, we examine the generation of the superoscillatory light waves with a smaller sample. In this case, the

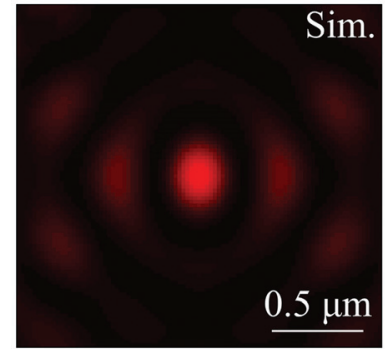

(a)

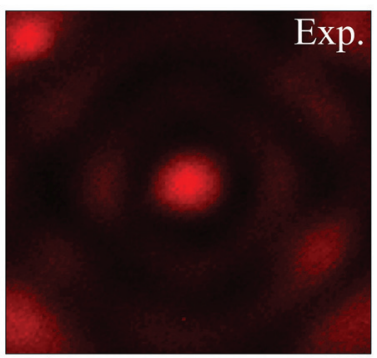

(c)

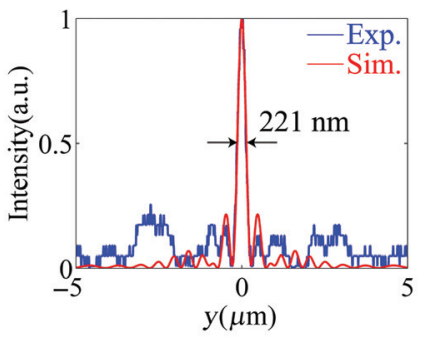

(e)

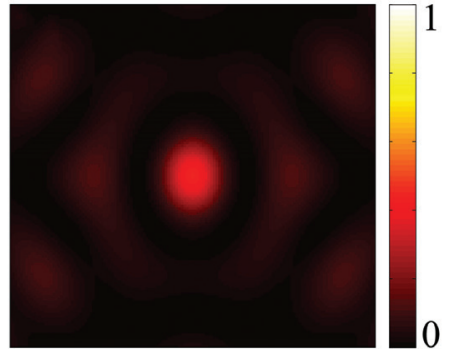

(b)

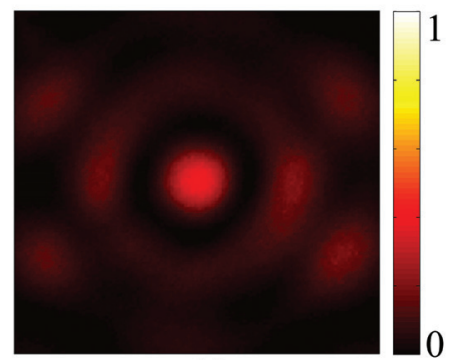

(d)

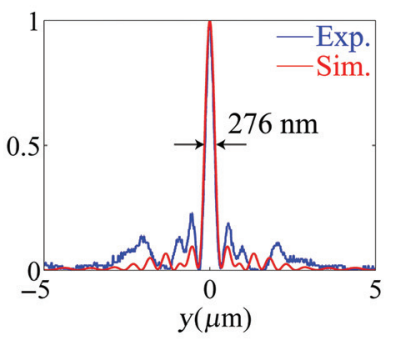

(f)
Fig. 4 Generation of the superoscillatory light waves with a smaller moonlike nanostructure $(d=8 \mu \mathrm{m}$ and $\omega=2 \mu \mathrm{m})$. (a), (b) The simulated intensity distributions of the diffractive fields at distances of (a) $z=2.9 \mu \mathrm{m}$ and (b) $z=4.0 \mu \mathrm{m}$. (c), (d) The corresponding experimental measurements of (a) and (b), respectively. (e), (f) Intensity profiles along the $y$ coordinate. The FWHM values of the profiles were measured as 221 and $276 \mathrm{~nm}$. Blue and red curves show the experiment and the simulation [based on Eq. (2)], respectively. Panels (a)-(d) share the same scale. The labeled FWHMs in (e) and (f) refer to the experimental data.

diameter of the central disc is decreased to $d=8 \mu \mathrm{m}$, while keeping other parameters unchanged. In spite of the change of sample size, significantly superoscillatory waves without the side lobe accompanied along the $y$ dimension can still be observed. Figure 4 shows, both numerically and experimentally, intensity distributions of the diffractive focusing main lobes at two distances: $z=2.9 \mu \mathrm{m}$ [Figs. 4(a), 4(c), and 4(e)], and $z=$ $4.0 \mu \mathrm{m}$ [Figs. 4(b), 4(d), and 4(f)]. As indicated from their corresponding profiles, the diffractive focal spots have no accompanying significant side lobes, and the sizes at FWHM are measured as 221 and $276 \mathrm{~nm}$, respectively, both below the diffraction limit. Although these figures show similar results to that displayed in Fig. 2, it is evident that increasing the diameter of the central disc would lead to lengthening the working distance of the superoscillatory light waves. For instance, with a small size $d=8 \mu \mathrm{m}$, significant superoscillatory waves with the feature size measured as $221 \mathrm{~nm}$ are observed at distance of $z=2.9 \mu \mathrm{m}$, as shown in Fig. 4(e); while for a large size $d=15 \mu \mathrm{m}$, the working distance increases to $z=5.5 \mu \mathrm{m}$, at which a comparable 
superoscillatory effect is observed, as shown in Fig. 3(d). These advantages of no side lobe and controllable working distance associated with the superoscillatory waves might be beneficial to far-field superresolution microscopy. ${ }^{2,16,19,28}$

Finally, we investigate free-space propagation of the generated superoscillatory light waves. We note that the propagation dynamics of the superoscillatory waves was considered previously. ${ }^{33,41-43}$ In this work, the generated superoscillatory wave results from superposition of the in-phase diffracted waves. Hence, the generated wave form has a particularly Bessel-like function that exhibits weakly diffracting property during propagation. $^{44,45}$ To this end, we performed experiments and recorded the intensity distributions of the light fields along the distances for both of the sample sizes: $d=8 \mu \mathrm{m}$ and $d=15 \mu \mathrm{m}$. To illustrate the propagation of the no-side-lobe superoscillatory waves along the symmetric cut, each cross section of the measured light fields along the $y$ coordinate is aligned in the $z$ axis, with the results illustrated in Figs. 5(b) and 5(d) for both cases of moonlike samples. Clearly, we observe that the superoscillatory light wave, indeed, does not change significantly its shape and travels along the distance in space for a long distance. The slight diffraction of the superoscillatory main lobe indicates that the delicate subwavelength feature can be maintained up to a critical distance $z_{c}$, where superoscillation disappears. For instance, for a small sample $(d=8 \mu \mathrm{m})$, it can be calculated as $z_{c}=6.0 \mu \mathrm{m}$; whereas for a larger one $(d=15 \mu \mathrm{m})$, the critical distance increases to $z_{c}=9.6 \mu \mathrm{m}$. The corresponding simulations in

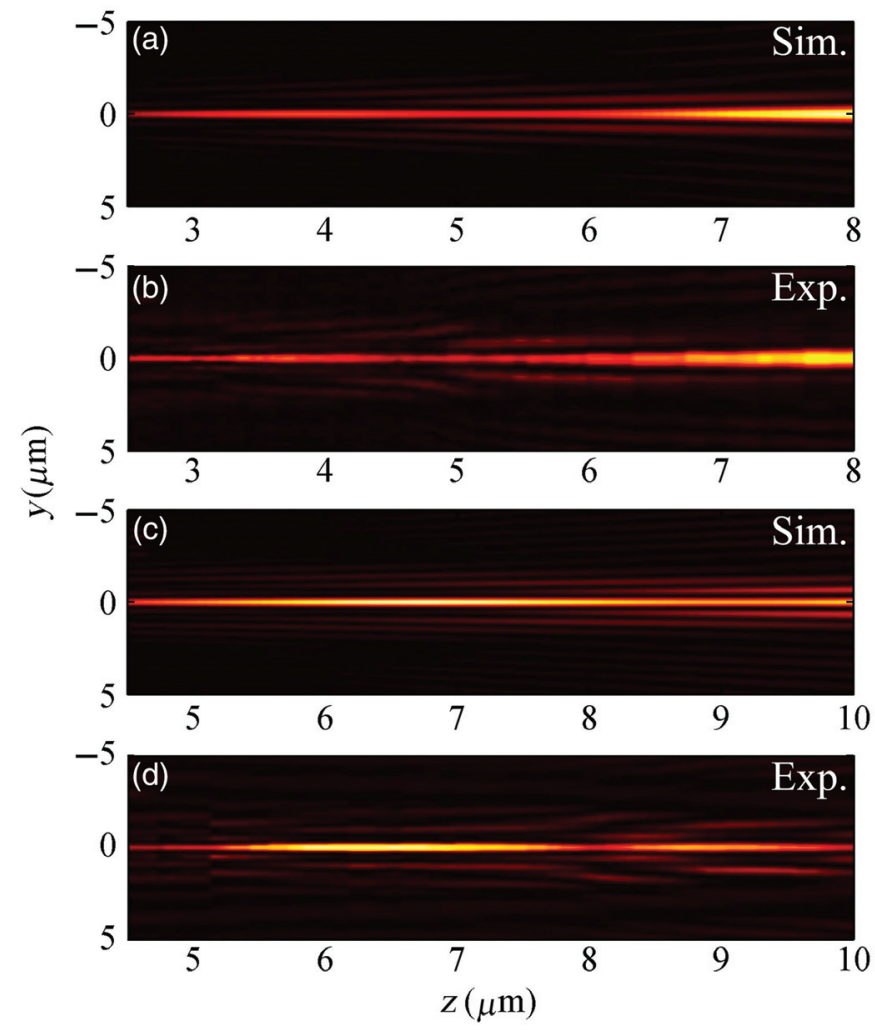

Fig. 5 Free-space propagation of the generated superoscillatory waves. The panels depict the measured intensity distributions of the light waves in the $y-z$ plane in the cases of (a), (b) $d=8 \mu \mathrm{m}$ and (c), (d) $d=15 \mu \mathrm{m}$. (a), (c) The simulated results based on Eq. (2), whereas panels (b) and (d) present the corresponding experiments.
Figs. 5(a) and 5(c) show good agreement with the experiments. We note that the weakly diffracting property of the light beams leads to a slight increase of the transverse size of the main lobes with distance. This can be explained by the resultant Bessel-like profile, which shows a function of distance $z,{ }^{36}$ different from the Bessel beams that show an invariant profile within the nondiffracting range. ${ }^{44,45}$ As a result, the superoscillatory phenomenon eventually disappears when increasing the distance. We also point out that the symmetric cut of the moonlike element would slightly break the cylindrical symmetry of the high-spatialfrequency wavevectors in the reciprocal space, which leads to slight intensity oscillation along the propagation distance, as shown in Fig. 5, both numerically and experimentally.

\section{Conclusions}

For achieving high spatial resolution, the intriguing features of superoscillatory waves provide promising advantages over other technologies. ${ }^{2,17,18}$ However, a critical issue when using the optical superoscillation is to eliminate the side lobes. To this end, we have implemented the concept of destructive interference of waves. Specifically, a new optical superoscillatory wave form without side lobes accompanied along a symmetric cut of the light pattern has been investigated both experimentally and theoretically. We realized it with a pair of moonlike apertures having sharp edges. Such an element permits generating an extremely localized wave packet with a feature size that can be far beyond the diffraction limit. In addition, the presented superoscillatory waves have Bessel-like forms, which allows for maintaining their deep-subwavelength structure to a farther distance than the evanescent waves. In comparison to the complex optimization-based SOLs, ${ }^{16,22-26}$ the proposed structure has advantages of no optimization and simplicity, i.e., the nanostructure can be achieved without any skill-based techniques. Therefore, our demonstrations may open new avenues to explore the concept of no-side-lobe superoscillation as well as relevant applications in various potential fields, e.g., it could be utilized for high-resolution optical microscopy, as significant improvement of spatial resolution in optical microscopic systems could be achieved using structured light beams such as Bessel beams, ${ }^{19}$ vector beams, ${ }^{6}$ and superoscillatory beams. ${ }^{2,16,19,28}$ It is worth mentioning that we can obtain the no-side-lobe superoscillatory light spot along a symmetric cut that, in principle, has arbitrary direction in the transverse plane. This can be achieved by rotating the moonlike apertures.

\section{Acknowledgments}

This work was partially supported by the National Natural Science Foundation of China (11974146, 61935010), the National Key Research and Development Program of China (2017YFB1104500), the Natural Science Foundation of Guangdong Province (2017B030306009, 2018B010114002), the Pearl River Talent Project (2017GC010280), and the Guangzhou Science and Technology Plan Project (201904010094).

\section{References}

1. E. B. zur Abbe, "Theorie des Mikroskops und der mikroskopischen Wahrnehmung," Arch. Mikroskop. Anat. 9(1), 413-468 (1873).

2. X. Hao et al., "From microscopy to nanoscopy via visible light," Light: Sci. Appl. 2(10), e108 (2013).

3. F. Lemoult et al., "Resonant metalenses for breaking the diffraction barrier," Phys. Rev. Lett. 104(20), 203901 (2010). 
4. R. Zuo et al., "Breaking the diffraction limit with radially polarized light based on dielectric metalenses," Adv. Opt. Mater. 6(21), 1800795 (2018).

5. B. Huang, H. Babcock, and X. Zhuang, "Breaking the diffraction barrier: super-resolution imaing of cells," Cell 143(7), 1047-1058 (2010).

6. X. Xie et al., "Harnessing the point-spread function for highresolution far-field optical microscopy," Phys. Rev. Lett. 113(26), 263901 (2014).

7. E. Betzig et al., "Breaking the diffraction barrier: optical microscopy on a nanometric scale," Science 251(5000), 1468-1470 (1991).

8. S. Thomas et al., "Probing of optical near-fields by electron rescattering on the $1 \mathrm{~nm}$ scale," Nano Lett. 13(10), 4790-4794 (2013).

9. N. Fang et al., "Sub-diffraction-limited optical imaging with a silver superlens," Science 308(5721), 534-537 (2005).

10. X. Zhang and Z. Liu, "Superlenses to overcome the diffraction limit," Nat. Mater. 7(6), 435-441 (2008).

11. J. B. Pendry, "Negative refraction makes a perfect lens," Phys. Rev. Lett. 85(18), 3966-3969 (2000).

12. Z. Liu et al., "Far-field optical hyperlens magnifying subdiffraction-limited objects," Science 315(5819), 1686 (2007).

13. S. W. Hell and J. Wichmann, "Breaking the diffraction resolution limit by stimulated emission: stimulated-emission-depletion fluorescence microscopy," Opt. Lett. 19(11), 780-782 (1994).

14. M. Bossi et al., "Breaking the diffraction resolution barrier in farfield microscopy by molecular optical bistability," New J. Phys. 8(11), 275-284 (2006).

15. F. M. Huang and N. I. Zheludev, "Super-resolution without evanescent waves," Nano Lett. 9(3), 1249-1254 (2009).

16. E. T. F. Rogers et al., "A super-oscillatory lens optical microscope for subwavelength imaging," Nat. Mater. 11(5), 432-435 (2012).

17. G. Gbur, "Using superoscillations for superresolved imaging and subwavelength focusing," Nanophotonics 8(2), 205-225 (2019).

18. G. Chen, Z. Wen, and C. Qiu, "Superoscillation: from physics to optical applications," Light: Sci. Appl. 8(1), 56 (2019).

19. Y. Kozawa, D. Matsunaga, and S. Sato, "Superresolution imaging via superoscillation focusing of a radially polarized beam," Optica 5(2), 86-92 (2018).

20. M. V. Berry and S. Popescu, "Evolution of quantum superoscillations and optical superresolution without evanescent waves," J. Phys. A: Math. Gen. 39(22), 6965-6977 (2006).

21. P. Woodward and J. Lawson, "The theoretical precision with which an arbitrary radiation-pattern may be obtained from a source of finite size," J. Inst. Electr. Eng. 95(37), 363-370 (1948).

22. D. Tang et al. "Ultrabroadband superoscillatory lens composed by plasmonic metasurfaces for subdiffraction light focusing," Laser Photonics Rev. 9(6), 713-719 (2015).

23. E. T. F. Rogers et al., "Super-oscillatory optical needle," Appl. Phys. Lett. 102(3), 031108 (2013).

24. K. Huang et al. "Optimization-free superoscillatory lens using phase and amplitude masks," Laser Photonics Rev. 8(1), 152-157 (2014).

25. G. Yuan, E. T. F. Rogers, and N. I. Zheludev, “'Plasmonics' in free space: observation of giant wavevectors, vortices, and energy backflow in superoscillatory optical fields," Light: Sci. Appl. 8(1), 2 (2019).

26. G. Yuan and N. I. Zheludev, "Detecting nanometric displacements with optical ruler metrology," Science 364(6442), 771-775 (2019).

27. G. T. Di Francia, "Super-gain antennas and optical resolving power," Nuovo Cimento Suppl. 9(S3), 426-438 (1952).

28. F. Qin et al. "A supercritical lens optical label-free microscopy: sub-diffraction resolution and ultra-long working distance," $A d v$. Mater. 29(8), 1602721 (2017)

29. B. K. Singh et al., "Particle manipulation beyond the diffraction limit using structured super-oscillating light beams," Light: Sci. Appl. 6(9), e17050 (2017).

30. X. H. Dong et al., "Superresolution far-feild imaging of complex objects using reduced superoscillating ripples," Optica 4(9), 1126-1133 (2017).
31. Y. Eliezer et al., "Breaking the temporal resolution limit by superoscillating optical beats," Phys. Rev. Lett. 119(4), 043903 (2017).

32. Y. Eliezer et al. "Experimental realization of structured superoscillatory pulses," Opt. Express 26(4), 4933-4941 (2018).

33. H. Lin et al. "Generation and propagation of optical superoscillatory vortex arrays," Ann. Phys. 531(11), 1900240 (2019).

34. Y. Hu et al., "Focusing optical waves with a rotationally symmetric sharp-edge aperture," Opt. Commun. 413, 136-140 (2018).

35. D. Weisman et al., "Diffractive focusing of waves in time and in space," Phys. Rev. Lett. 118(15), 154301 (2017).

36. G. D. Gillen and S. Guha, "Modeling and propagation of nearfield diffraction patterns: a more complete approach," Am. J. Phys. 72(9), 1195-1201 (2004)

37. T. Roy et al., "Point spread function of the optical neddle superoscillatory lens," Appl. Phys. Lett. 104(23), 231109 (2014).

38. H. Ni et al. "Large-scale high-numerical-aperture super-oscillatory lens fabricated by direct laser writing lithography," RSC $A d v$. 8(36), 20117-20123 (2018).

39. G. Yuan et al. "Far-field superoscillatory metamaterial superlens," Phys. Rev. Appl. 11(6), 064016 (2019).

40. G. Yuan, E. T. F. Rogers, and N. I. Zheludev, "Achromatic superoscillatory lenses with subwavelength focusing," Light: Sci. Appl. 6(9), e17036 (2017).

41. E. Greenfield et al., "Experimental generation of arbitrarily shaped diffractionless superoscillatory optical beams," Opt. Express 21(11), 13425-13435 (2013).

42. Y. Eliezer and A. Bahabad, "Super-oscillating airy pattern," ACS Photonics 3(6), 1053-1059 (2016).

43. T. Zacharias et al. "Axial sub-Fourier focusing of an optical beam," Opt. Lett. 42(16), 3205-3208 (2017).

44. J. Durnin, J. J. Miceli, Jr., and J. H. Eberly, "Diffraction-free beams," Phys. Rev. Lett. 58(15), 1499-1501 (1987).

45. D. Mcgloin and K. Dholakia, "Bessel beams: diffraction in a new light," Contemp. Phys. 46(1), 15-28 (2005).

Yanwen $\mathrm{Hu}$ is currently a $\mathrm{PhD}$ graduate student with Professor Zhenqiang Chen at Jinan University, China. His research interest is subwavelength generations of light beams and their applications.

Shiwang Wang is an engineer in optical design at Sunny Optics (Zhongshan) Co., Ltd. He received his MS degree from Jinan University in 2020 .

Junhui Jia is a master student with Professor Shenhe Fu at Jinan University. His main research interest is to study wave phenomenon in light-matter interaction process.

Shenhe $\mathbf{F u}$ is currently a professor in the Department of Optoelectronic Engineering, Jinan University, Guangzhou, China. He received his $\mathrm{PhD}$ from Sun Yat-sen University. His research focuses on light-field manipulations and applications.

Hao Yin is currently a professor in the Department of Optoelectronic Engineering, Jinan University, Guangzhou, China. He received his PhD from Jinan University. His research interest is on laser technologies.

Zhen Li is currently a professor in the Department of Optoelectronic Engineering, Jinan University, Guangzhou, China. He received his PhD from Sun Yat-Sen University. His research mainly focuses on laser physics and laser technologies.

Zhenqiang Chen is currently a professor in the Department of Optoelectronic Engineering, Jinan University, Guangzhou, China. He received his $\mathrm{PhD}$ from China University of Geosciences. His research interests include laser technologies, laser physics, laser materials, and laser applications. 